\title{
A MEMS 2-D Scanner with Bonded Single-Crystalline Honeycomb Micromirror
}

\author{
Pamela R. Patterson, Guo-Dung J. Su, Hiroshi Toshiyoshi, Ming C. Wu \\ Electrical Engineering Department, University of California, Los Angeles \\ Los Angeles, Ca 90095-1594
}

\begin{abstract}
We report on the fabrication and performance of a surfacemicromachined 2-D scanner with bonded single-crystalline honeycomb micromirror. The silicon honeycomb is derived from silicon on insulator (SOI) and resolves curvature problems seen in thin film polysilicon mirrors, without incurring the mass increase of a solid silicon structure. Honeycomb micromirrors with surface roughness of $<12 \mathrm{~nm}$ and curvature of $143 \mathrm{~cm}$ have been demonstrated. The 2D scanner is electrostatically actuated and has a mechanical scan angle of $6^{\circ}$. Optical performance of the bonded honeycomb micromirror is comparable to that of solid silicon mirrors.

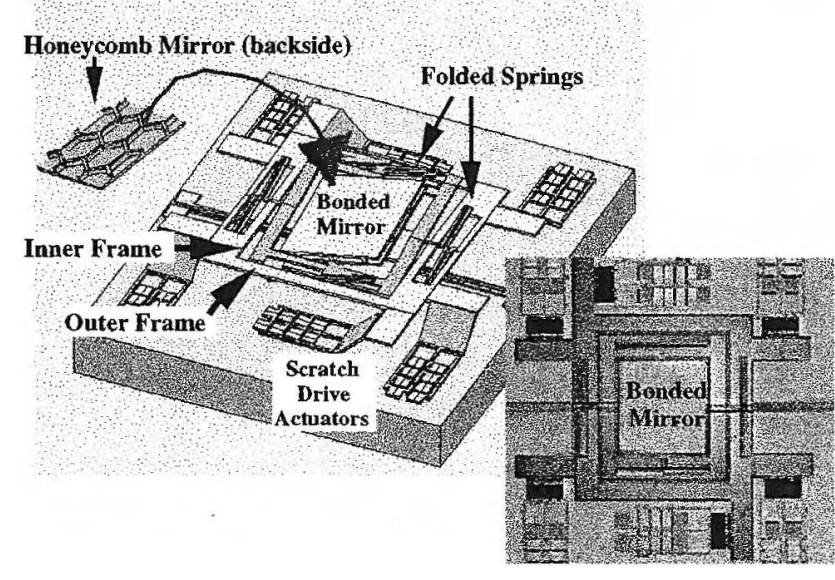

Figure 1. Schematic rendering and photograph of polysilicon $2 D$ scanner with bonded silcon honeycomb mirror.

\section{INTRODUCTION}

Fast steering mirrors are used in many applications, including display, switching, sensing, and optical data storage [1]. Surfacemicromachining technology paves the way for the design and fabrication of compact, lightweight scanners in a production mode similar to the planar processing of integrated circuits. However, surface-micromachined structures, i.e., released thin films, are extremely sensitive to residual stress and stress gradient, as can be seen in the significant curvature of standard polysilicon micromirrors [2]. This curvature degrades the resolution of the scanning micromirror.

Several approaches have been proposed to reduce the curvature of micromirrors [3]. We have recently reported on a surface-micromachined 2-D scanner with a flat, smooth, single crystalline micromirror formed from SOI [4]. This approach is compatible with the standard surface-micromachining process. While this device shows orders of magnitude improvement in optical properties, the mass of the scanner is increased. To maximize the resonant frequency, a reduction of the total mass is desirable.
In this paper, we report on the successful fabrication of a 2-D scanner with an optically flat, lightweight, honeycomb micromirror. The honeycomb core is a well know structural element for optimization of lightweight and strength. Microhoneycombs have been used as molds for MEMS structures $[5,6]$. We employ deep reactive ion etching (DRIE) and wafer bonding techniques to fabricate single-crystalline honeycomb micromirrors. Smooth mirror surface $(11.5 \mathrm{~nm})$ and large radius of curvature (143 $\mathrm{cm})$ are achieved. The 2-D scanner has a mechanical scan angle of $6^{\circ}$

\section{DESIGN AND FABRICATION}

The schematic and photograph of the 2-D scanner with honeycomb micromirror are shown in Fig. 1. The electrostatic actuators of the scanner are fabricated by standard polysilicon surface-micromachining process (MUMPs) [7]. The honeycomb mirrors are formed on SOI wafers with $23-\mu \mathrm{m}$-thick top layers. The SOI substrates were first thinned down to $200 \mu \mathrm{m}$ by mechanical lapping. The honeycomb pattern (hexagonal cells with $100-\mu \mathrm{m}$-long sides and $10-\mu \mathrm{m}$-thick walls) was etched into the top surface silicon using DRIE. The etch was stopped before reaching the buried oxide layer such that a faceplate of silicon remained. After formation of the honeycomb structure, the SOI wafer was bonded to the actuator wafer with patterned photoresist as adhesive in a vacuum oven at $140^{\circ} \mathrm{C}$. The SOI substrate was subsequently removed by DRIE etch with the oxide layer providing an etch stop. The oxide was removed in an HF solution prior to honeycomb mirror alignment and patterning. The honeycomb mirrors with $450 \mu \mathrm{m} \times 450 \mu \mathrm{m}$ areas were aligned to the polysilicon actuators by photolithography and etched using DRIE. The exposed photoresist was removed in oxygen plasma. A hard bake at $230^{\circ} \mathrm{C}$ was done before release of the polysilicon scanner in $49 \% \mathrm{HF}$. Finally, the bonded scanning mirror was assembled and raised to $84 \mu \mathrm{m}$ above the substrate using the on-chip actuators [7].

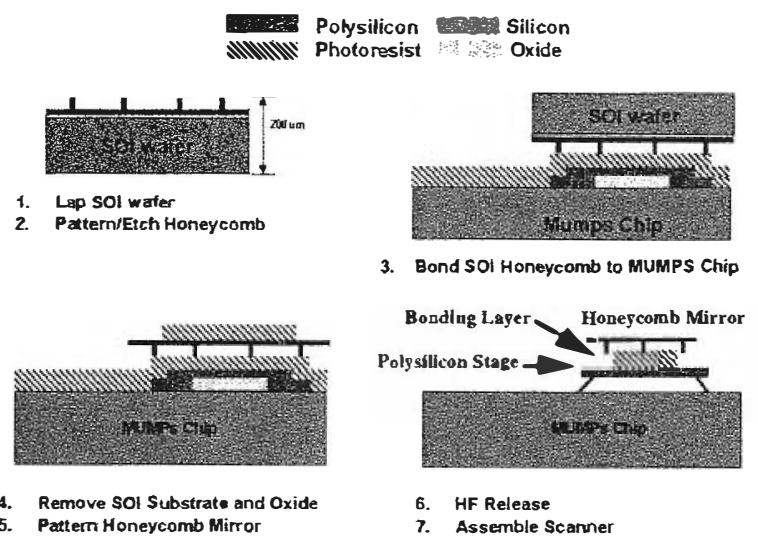

Figure 2. Fabrication process flow 


\section{MEASUREMENT RESULTS AND DISCUSSION}

The etched profiles of the honeycomb structures are shown in Figure 3 for two different etched depths. The measurements were made using a WYKO RTS 500 surface profiler. Honeycomb samples with faceplate thickness of $1.3,9$, and $12 \mu \mathrm{m}$ have been made.
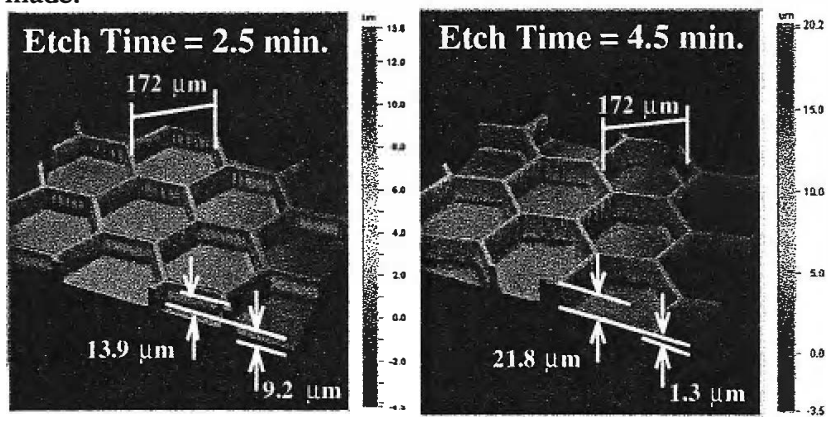

Figure 3. Honeycomb-etched SOI showing depth vs. DRIE etch time
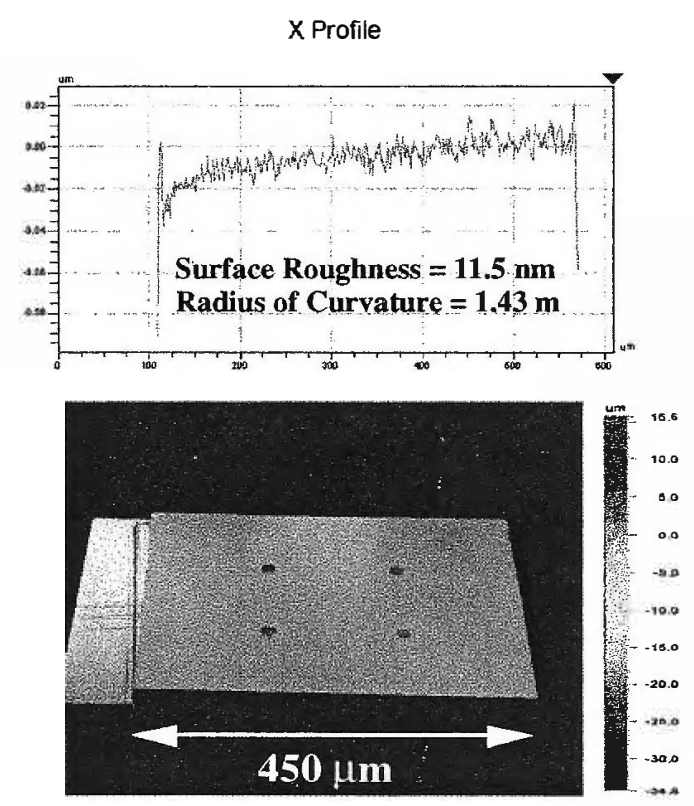

Figure 4. Cross section profile and $3 D$ plot of bonded honeycomb mirror

We have also measured the surface quality of the bonded honeycomb mirrors. The surface roughness values are 123, 12, and $11 \mathrm{~nm}$ for honeycomb micromirrors with faceplate thickness of 1.3 , 9 , and $12 \mu \mathrm{m}$, respectively. The corrugation seen in the $1.3-\mu \mathrm{m}$ thick sample is most likely due to the current bonding process. We are working on alternative methods that will allow us to realize a smooth surface, for faceplate thickness to $\sim 1 \mu \mathrm{m}$. It should be noted that even with the current corrugation, the surface roughness is still less than $\lambda / 10$ at $1.55 \mu \mathrm{m}$ wavelength, which is sufficient for most applications.

Far Field images of reflected light were measured with a collimated $\mathrm{He}-\mathrm{Ne}$ laser on the $9 \mu \mathrm{m}$ sample. This image is essentially identical to the reference wafer, and is significantly improved from the polysilicon micromirrrors. The WYKO measurements for the bonded mirror with a $12 \mu \mathrm{m}$ faceplate show the minor is flat with a large radius of curvature $(143 \mathrm{~cm})$. The transfer characteristics of the scanner is measured with a Polytech laser Doppler vibrometer. The mechanical scan angle was found to be $6^{\circ}$ at $50 \mathrm{~V}$.

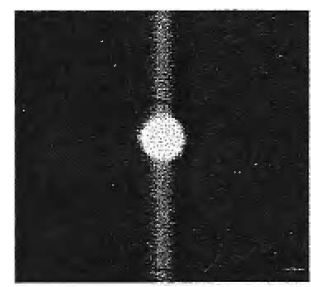

Silicon Ref. wafer

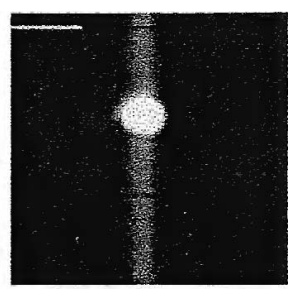

Honeycomb bonded silicon wafer

\section{Figure 5. Far field images of reflected $\mathrm{He}$-Ne laser light}

\section{CONCLUSION}

We have proposed and demonstrated a new technique to fabricate flat, lightweight honeycomb micromirrors for surfacemicromachined 2-D scanners. Honeycomb micromirrors with surface roughness $<12 \mathrm{~nm}$ and a radius of curvature of $143 \mathrm{~cm}$ have been demonstrated. 2-D optical scanners with $12^{\circ}$ optical scan angle and diffraction-limited optical performance have been achieved.

\section{ACKNOWLEDGEMENTS}

The authors would like to thank Allan Hui and Hung Nguyen for technical assistance. The project is supported in part by DARPA.

\section{REFERENCES}

1. Muller, R.S.; Lau, K.Y, "Surface-micromachined microoptical elements and systems," Proc. IEEE, vol. 86, p.1705, 1998.

2. R.A. Conant, P.M. Hagelin, U. Krishnamoorthy, O. Solgaard, K.Y. Lau, R.S. Muller, "A full-motion video display using micromachined scanning micromirrors," Proc. Transducers '99, Sendai, Japan, June 1999, pp. 376-379.

3. J. Nee, R. Conant, M. Hart, R. Muller, K. Lau, "Stretched-film micromirrors for improved optical flatness,” MEMS 2000, pp. 704 709.

4. G. J. Su, H. Nguyen, P. Patterson, H. Toshiyoshi and M.C.Wu, "Surface-Micromachined 2D Optical Scanners with HighPerformance Single-Crystalline Silicon Micromirrors", to be published in CLEO 2000.

5. W.Ehrfeld, P.Bley, F.Gotz, P. Hagmann, A.Maner, J. Mohr, H.O.Moser,D.Munchmeyer,W.Schelb,D.Schmidt,E.W.Baker,

"Fabrication of Microstructures using the Liga Process", Proc. IEEE Micro Robots and Teleoperators Workshop, Hyannis, MA, November 1987, pp.11/1-11/11.

6. C. Keller, and M. Ferrari, "Milli-Scale Polysilicon Structures", 1994 Solid-State Sensor and Actuator Workshop, Hilton Head, pp. 132 - 137.

7. L. Fan, M. Wu, K. Choquette, and M. Crawford, "Selfassembled microactuated XYZ stages for optical scanning and alignment", Transducers '97, pp. 319-322. 